\title{
The role of Continuous Positive Airway Pressure (CPAP) in Delaying or Avoiding Mechanical Ventilation in COVID-19 Respiratory Failure: A Retrospective Cohort Study
}

\author{
Allan Cameron \\ Glasgow Royal Infirmary \\ Sharif Fattah ( $\square$ sharif.fattah@ggc.scot.nhs.uk) \\ Glasgow Royal Infirmary https://orcid.org/0000-0002-1781-5746 \\ Laura Knox \\ Glasgow Royal Infirmary \\ Pauline Grose \\ Glasgow Royal Infirmary
}

Research

Keywords: COVID-19, CPAP, Respiratory Failure

Posted Date: August 5th, 2021

DOI: https://doi.org/10.21203/rs.3.rs-722133/v1

License: (1) (1) This work is licensed under a Creative Commons Attribution 4.0 International License.

Read Full License 


\section{Abstract}

Background - During the winter of 2020-2021, the second wave of the COVID19 pandemic in the United Kingdom caused increased demand for intensive care unit (ICU) beds, and in particular, for invasive mechanical ventilation (IMV). To alleviate some of this pressure, some centres offered non-invasive continuous positive airway pressure (CPAP), delivered on specialised COVID high dependency units (cHDUs). However, this practice was based largely on anecdotal reports, and it is not clear from the literature how effective CPAP is at delaying or preventing IMV.

Methods - This was a retrospective observational cohort study of consecutive patients admitted to a specialised cHDU at Glasgow Royal Infirmary between November 2020 and February 2021. Each patient had a continuous record of the level of respiratory support required, and was followed up to hospital discharge or death. We examined patient outcomes according to age, sex and maximum level of respiratory support, using logistic regression and time-to-event analysis. The number of patients who could not be oxygenated by standard oxygen facemask but could be oxygenated by CPAP was counted and compared to the number of patients admitted to ICU for IMV over the same period.

Results - There were 152 admissions to cHDU over the study period. Of these, 125 received CPAP treatment. Of the patients who received support in $\mathrm{CHDU}$, the overall mortality rate was $37.9 \%(95 \% \mathrm{Cl}$ $30.3 \%-46.1 \%)$ ). Odds of mortality were closely correlated with increasing age and oxygen requirement. Of the 152 patients, 44 patients $(28.8 \%, 95 \% \mathrm{Cl} 22.0-36.9 \%)$ went on to require IMV in ICU. This represents $77.2 \%$ of the 57 COVID-19 admissions to ICU during the same period. However, there were also 41 patients who received levels of respiratory support on cHDU which would normally necessitate ICU admission but who never went to ICU, potentially reducing ICU admissions by $41.8 \%(95 \% \mathrm{Cl} 32.1-$ $52.2 \%)$.

Conclusion - Providing respiratory support in cHDU reduced the number of potential ICU admissions by $41.8 \%$, as well as delaying IMV for over $75 \%$ of ICU admissions. This represents a significant sparing of ICU capacity at a time when IMV beds were in high demand.

\section{Introduction}

The SARS-Cov-2 pandemic has caused significant morbidity and mortality worldwide, being responsible for more than 3 million deaths globally as of May $2021^{[1]}$. Despite the recent development of therapies shown to reduce mortality and the rollout of vaccination programmes against COVID-19, global case numbers, hospitalisations and deaths remain high ${ }^{[1-3]}$.

The large number of patients with COVID-19 has put pressure on many health services, and in particular a high demand for critical care beds; at the peak of the second COVID-19 wave in the UK in January 2021, there were over 4,000 patients on invasive mechanical ventilation (IMV) for COVID-19 across the country [4]. 
Beginning in March 2020, some National Health Service (NHS) resources were diverted to help support intensive care units (ICUs) in preparation for the anticipated demand. In several centres, this included the development of respiratory support units (RSUs) and COVID-19 high dependency units (cHDUs), as areas where patients could receive non-invasive respiratory support such as continuous positive airway pressure (CPAP) and high flow nasal oxygen (HFNO). Both of these modalities are considered aerosolgenerating procedures (AGPs), and hence must be provided in specific facilities with stringent infection control procedures and specialized staff. CPAP and HFNO are used for a variety of reasons in COVID-19, but one rationale is to reduce the number of patients requiring IMV at any one time ${ }^{[5-7]}$.

CPAP has been used previously in pneumonitis caused by other coronaviruses, playing a role in the treatment of Severe Acute Respiratory Syndrome (SARS) and Middle East Respiratory Syndrome (MERS)

$[8,9]$. Its utilization in COVID-19 was also described in hospitals in China and Italy at the start of the pandemic ${ }^{[10]}$. This led the British Thoracic Society to recommend CPAP as first-line respiratory support in patients with COVID-19 pneumonitis who fail to oxygenate adequately on standard oxygen therapy ${ }^{[11]}$.

CPAP typically uses less oxygen than high-flow nasal oxygen therapy, and thus places less of a burden on a hospital's oxygen supply when the hospital has a high COVID-19 workload. Due to it being a noninvasive modality, CPAP has the potential to act as both a bridge to delay or avoid IMV, and as a ceiling of care for those unsuitable for IMV.

To our knowledge, the extent to which a dedicated CPAP unit can relieve the demand for ICU beds has not been well described in the literature.

\section{Objectives}

The aim of this study is to describe the outcomes of a cohort of patients with COVID-19 pneumonitis treated in a dedicated COVID-19 high dependency unit. In particular, we examine whether use of CPAP or HFNO therapy could delay or avoid IMV.

\section{Methods}

\section{Study Design}

This was a retrospective cohort study of consecutive patients admitted to the cHDU at Glasgow Royal Infirmary over a three-month period coinciding with the second wave of Coronavirus in the UK between November 2020 and February 2021.

\section{Setting}

The Glasgow Royal infirmary (GRI) is a large 950-bedded city teaching hospital serving a population of around 320,000 people. The city as a whole had a high prevalence of COVID-19 during the study period with a peak 7-day rate of cases per 100,000 population of 402 at the beginning of January $2021^{\text {[12] }}$. 
During the first wave of the pandemic in March 2020, a 14-bedded COVID-19 high dependency unit (cHDU) was established at GRI. By the time this was reestablished for the second wave of COVID-19 in Autumn 2020, staff had already developed considerable experience in managing COVID-19 with CPAP and HFNO, and there were agreed referral and treatment pathways already in place. Dexamethasone had been introduced as an evidence-based treatment, and there was emerging evidence for the use of other therapeutics such as Remdesevir and Tocilizumab.

The unit's facilities included a 2:1 patient-to-nurse ratio; the ability to provide continuous invasive monitoring when indicated; 24-hour senior-level medical presence, and appropriate physical barriers around the ward so that aerosol generating procedures (AGPs) could take place in a sealed environment. Staff wore full personal protective equipment including FFP3 respirator masks to minimise exposure.

CPAP was provided via face mask using ResMed Stellar ${ }^{\text {rM }}$ machines with supplemental oxygen administered via a wall-mounted flow valve capable of 30L/min delivery. We used a standard PEEP of 10 $\mathrm{cmH}_{2} \mathrm{O}$ for all patients on CPAP, and all patients treated with CPAP were given $5 \mathrm{mg}$ midzolam and $5 \mathrm{mg}$ morphine over 24 hours by subcutaneous infusion to improve mask tolerance unless specifically contraindicated.

In-house testing of the $\mathrm{FiO}_{2}$ achieved at various oxygen flow rates was undertaken by our Respiratory Physiology Colleagues, this is summarised in Table 1.

\begin{tabular}{|c|c|}
\hline Oxygen & FiO.2. \\
\hline $10 \mathrm{l} / \mathrm{min}$ & $40-45 \%$ \\
\hline $15 \mathrm{l} / \mathrm{min}$ & $50 \%$ \\
\hline $20 \mathrm{l} / \mathrm{min}$ & $60-70 \%$ \\
\hline $25 \mathrm{l} / \mathrm{min}$ & $80 \%$ \\
\hline $30 \mathrm{l} / \mathrm{min}$ & $75-90 \%$ \\
\hline
\end{tabular}

NHFO was provided by the Fisher and Paykel AIRVO $2^{\text {TM }}$, which on maximum settings provided $60 \mathrm{~L} / \mathrm{min}$ of warmed, humidified gas with an $\mathrm{FiO}_{2}$ of $95 \%$.

Oxygenation was assessed by pulse oximetry, and the oxygenation target was $90-94 \%$ unless the patient was known to have chronic hypoxia from a pre-existing condition, in which case the target was $88-92 \%$.

\section{Patients}


We examined records for all consecutive adult patients admitted to the cHDU at Glasgow Royal Infirmary between 15th November 2020 and 14th February 2021 inclusive. Admission to the unit was the sole eligibility criterion. The start date was chosen for convenience, as this was when daily electronic recording of observations began on the unit, and the end date was chosen because the number of new admissions had fallen to low levels.

\section{Variables}

The data extracted from electronic records included each patient's age, sex and NCoV-19 PCR status. We recorded dates and times of admission to hospital, admission to cHDU, and where appropriate, admission to ICU. Each patient's date of discharge from hospital (or date of death) was also obtained. For each patient, a daily record of various parameters measured during their time in cHDU was obtained. This included respiratory rate and $\mathrm{SpO}_{2}$, type of respiratory support, $\mathrm{FiO}_{2}$ and $\mathrm{PEEP}$.

Our main outcome variables were the binary outcomes of ICU admission and of death. The explanatory variables were type of respiratory support, amount of supplemental oxygen, and duration of respiratory support. Age and sex were considered as important confounding variables.

\section{Data sources}

Patients were identified and cross-checked using the WardWatcher and TrakCare ${ }^{\mathrm{TM}}$ patient information systems, which also provided dates and times of admissions, discharges and deaths. The patient observations and respiratory settings were extracted from the TURAS COVID-19 application, which allowed electronic recording of each patient's parameters throughout their cHDU stay. Each TURAS daily review (and initial TURAS assessment, where available) was obtained in Portable Document Format (PDF) from Orion Software's Clinical Portal ${ }^{\mathrm{TM}}$ and the relevant data extracted from the PDF file using the pdftools package in $\mathrm{R}^{[13]}$. Missing data was rectified by review of electronic scans of written clinical notes available as PDFs on Clinical Portal.

\section{Bias}

Since this was an observational study, there will be unmeasured confounding variables. The most important are likely to be patient-level variables such as co-morbidities and frailty, which would affect the decision to admit to ICU, and make adverse outcomes more likely. However, there may also be a timedependent patient selection bias, as bed availability dropped during the peak of the wave in January, meaning that patients admitted during this time may well have been more unwell as the threshold for admission to $\mathrm{cHDU}$ increased. For the same reason, there may have been a time-dependent changing of the threshold for admission to ICU. There is no straightforward way to measure or correct for these potential biases, and they simply need to be borne in mind when interpreting the results.

\section{Study size}

Our study size was determined by the demand for and capacity of the unit during the study period. This allowed us to capture the majority of patients treated in the second wave of the coronavirus pandemic. 


\section{Quantitative variables}

The daily data for each patient was summarized to give a duration of time spent on respiratory support, and a maximum $\mathrm{FiO}_{2}$ or oxygen flow rate during their stay. To help give a more complete summary of the respiratory support on a per-patient basis, we also recorded the number of days spent on a high level of non-invasive respiratory support. This was defined as being on greater than $15 \mathrm{~L} / \mathrm{min}$ of oxygen whilst on $\mathrm{CPAP}$ or being on $\mathrm{HFNO}$ with an $\mathrm{FiO}_{2}$ of $\geq 0.9$. These numbers were chosen because by consensus they represented levels of support beyond that which could be achieved by standard oxygen therapy.

\section{Statistical methods}

Data was manually tidied and verified before being converted to comma separated variable format and imported into R version 4.1.0 [14]. Simple summary statistics including means, medians, ranges and histogram plots were produced using base $\mathrm{R}$ functions. Confidence intervals for simple proportions were calculated using binomial confidence intervals ${ }^{[15]}$. Comparison of proportions in two groups was done using Fisher's exact test. Comparisons of medians between two groups was performed using the MannWhitney $U$ test. Age density plots were created using normal kernel density estimates. The effect of binary outcomes according to continuous variables such as age or peak supplemental oxygen was carried out by logistic regression analysis. Kaplan-Meier curves were also constructed to perform survival analysis between men and women.

There was no missing data, as we were able to reconstruct missing data points from the written clinical notes.

To ensure reproducibility and transparency of the results, the statistical analysis code and methods section of this paper were written in R Markdown prior to the final data set becoming available.

\section{Results}

\section{Participants}

There were 1045 observations found for 153 episodes in 152 unique patients. The episodes comprised 71 admissions of women and 82 admissions of men.

The median age was 60 , with a range of $24-87$, and an interquartile range of $53-68$. The age distribution is shown more completely in the histogram in Fig. 1.

A total of 58 episodes ended in death, giving a crude mortality rate of $37.9 \%$ (95\% confidence interval $30.3 \%-46.1 \%)$ per admission.

The median length of stay in hospital was 11.8 days, with a range of $0.2-115$ days and interquartile range of $8-18.5$ days. 
The mean age of those who survived was 55.7, whereas those who died had a mean age of 65.1 ( $p<$ 0.001 by Mann-Whitney). Survivors were therefore around 10 years younger than non-survivors on average. The relative age distribution of survivors and non-survivors is depicted in Fig. 2.

\section{Association between age and mortality}

Age had a significant association with mortality. Logistic regression (Fig. 3) showed that the odds of death approximately doubled for every extra 8.0 years of age, with the survival odds being 1 (i.e. $50 \%$ probability of survival) at an age of 66.5 years.

\section{Association between sex and mortality}

31 out of the 71 women died (43.7\%) versus 27 of the 82 men (32.9\%). This difference was nonsignificant by Chi Square test $(p=0.231)$.

A comparison of the Kaplan Meier survival curves for men and women is shown in Fig. 4, and does appear to show a difference, with the hazard ratio for men being 0.606 , but the effect size just misses statistical significance $(\mathrm{Cl} 0.352-1.044, \mathrm{p}=0.071)$. This effect does not appear to be attributable to age, as there was less than 3 months' difference between the mean age of men and women in the cohort.

\section{ICU admission and mortality}

Of the patients who died after admission to cHDU, 27 of these died in ICU. The other 31 died either in cHDU or in a step down ward.

\section{Use of CPAP and HFNO}

142 patients had either continuous positive airway pressure (CPAP) applied via a facemask, or had high flow nasal oxygen (HFNO) for at least part of their time in cHDU.

The predominant modality was CPAP, with 95 (62.1\%) patients receiving CPAP only as their main modality. Only 17 (11.1\%) patients had HFNO only, with 30 (19.6\%) switching between modalities for various reasons, and $11(7.2 \%)$ not receiving either.

Of the 11 patients who did not receive an AGP, four patients were Covid positive with complex HDU needs other than respiratory support. Four patients had been stepped down from ICU, two were thought to require an AGP but stabilized quickly on admission and never required one. There was also one patient who turned out not to have Covid at all.

These findings are summarised in Table 2. 
Table 2

Modes of respiratory support used

\begin{tabular}{|llll|}
\hline $\begin{array}{l}\text { CPAP only } \\
(+/ \text { - HFNO for breaks })\end{array}$ & HFNO only & $\begin{array}{l}\text { Switched between } \\
\text { CPAP and HFNO } \\
\text { as primary device }\end{array}$ & $\begin{array}{l}\text { Had neither } \\
\text { HFNO nor CPAP }\end{array}$ \\
\hline 95 & 17 & 30 & 11 \\
\hline
\end{tabular}

\section{Association between level of respiratory support and survival}

Of the 125 patients who received CPAP at some point during their stay, there was a strong correlation between the need for maximum supplemental oxygen (30 litres per minute) and subsequent mortality. Only six out of 41 patients who required 30 litres per minute of supplemental oxygen with CPAP survived, a survival rate of $14.6 \%$. Of those who survived after reaching 30 litres, there were two patients who did not receive treatment in ICU. These both reached 30 litres for relatively short periods.

Conversely, patients who required less than 30 litres per minute had an overall survival rate of $81.0 \%$, though only nine patients reached a peak of between 20 and 30 litres per minute and only five (55.6\%) of them survived. The distribution of peak oxygen requirements, and their associated outcomes, is shown in Fig. 5.

This can be shown in percentage terms to get a better feel for the survival rate according to peak oxygen requirement (Fig. 6).

Again, we can perform a regression of peak supplemental oxygen with survival to show this correlation. The result is shown in Fig. 7.

We can see that a peak oxygen requirement of above 21 litres per minute makes death the most likely outcome. The log odds of death increase by 0.16 per litre of oxygen required, amounting to a doubling of the odds of death for every extra 4.2 litres of oxygen required per minute $(p<0.001)$.

A similar pattern was seen with patients on $\mathrm{HFNO}$. Those with a peak $\mathrm{FiO}_{2}$ below 0.9 had a survival rate of $72.7 \%$, whereas above 0.9 the survival rate fell to $41.7 \%$ (Fig. 8 ).

If we consider high respiratory support as being an $\mathrm{FiO}_{2}$ of 0.9 or more or CPAP with more than 15 litres of supplemental oxygen, then we can further examine the outcomes based on the number of days that a patient spent on high respiratory support (Fig. 9), where we can see that those with the greatest time on high respiratory support had the worst outcomes.

\section{ICU transfers and outcomes}

44 patients $(28.8 \%, \mathrm{Cl} 21.9 \%-36.7 \%)$ went to ICU. Of these, $17(38.6 \%)$ survived. 
Of these 17 ICU survivors, in two cases, the Covid swab was negative and the final diagnosis was not Covid pneumonitis. A further three patients had radiological evidence of bacterial pneumonia in addition to Covid at the time of their admission to ICU.

This means that 12 patients out of the $39(30.8 \%)$ patients with uncomplicated severe Covid pneumonitis survived their ICU admission. Even among this group, there is significant residual morbidity. One patient went home on domicillary oxygen; another with a Zimmer frame for mobility. One patient has since been readmitted with right heart failure due to post-Covid pulmonary fibrosis. Most have community rehab needs and some require ongoing community mental health input.

\section{Total ICU admissions during the study period and ICU avoidance}

There were 57 admissions in total to our ICU during the study period. These comprised 53 new admissions and four re-admissions. Our ICU were heavily pressured during the majority of this period, with full occupancy plus expansion beds opened elsewhere in the hospital. During this time, there were 41 patients treated in cHDU with levels of respiratory support exceeding the threshold that would normally mandate a transfer to ICU for IMV - that is, being on CPAP plus more than 15 litres per minute of oxygen, or being on $\mathrm{HFNO}$ with greater than $90 \% \mathrm{FiO}_{2}$. These are patients who typically could not be oxygenated on a ward without NIV or IMV. Although some of these patients may not have been deemed appropriate for IMV in ICU, this sets an upper limit of a $42 \%$ reduction in ICU admissions during the study period as a result of the facilities available in cHDU.

Although these patients are likely to have had a shorter length of stay in ICU than those who were actually taken for IMV, this still represents a large reduction in the burden placed on ICU resources.

\section{ICU admissions and maximal CPAP}

None of the 22 patients who were on the maximal 30 litres per minute of oxygen for more than 24 hours survived without going to ICU. Of the 14 patients on maximum CPAP for more than 24 hours who were taken to ICU, none who were on maximum CPAP for more than 3 days survived. There is likely an element of selection bias here, and the numbers are small, but in our cohort it seems that if a patient was on maximum CPAP for more than 24 hours, the prognosis was grim.

\section{Complications}

Six of the patients treated with CPAP (5\%) developed a pneumomediastinum, and one of these cases was associated with a small pneumothorax. Although two of these patients subsequently died, the pneumomediastinum was not thought to be a contributory cause. However, when pneumomediastinum or pneumothorax was identified in any of these patients, CPAP was discontinued and HNFO was used as an alternative. The increased incidence of pneumomediastinum in mechanically ventilated patients has already been discussed in the literature, and there are case reports of patients with COVID-19 pneumonitis 
developing pneumomediastinum even without IMV or NIV, so it is not clear that CPAP was the direct cause of these complications $[16,17]$.

\section{Discussion Key results}

Admissions to cHDU in this cohort were almost all appropriate candidates, meaning that the majority required some form of respiratory support beyond standard oxygen therapy. Predominantly these patients were brought for CPAP to treat COVID pneumonitis. Out of 153 patients, only two did not have a diagnosis of COVID pneumonitis.

Over $80 \%$ of patients received CPAP during their time in cHDU and less than $10 \%$ did not require an AGP. Only seven patients brought for respiratory support did not require an AGP. Four other patients were brought appropriately for complex care needs and did not receive an AGP. This suggests an appropriate use of the facility during the second wave of the pandemic.

The strongest clinical predictor of death was age, with the odds of a patient dying approximately doubling for every eight years of age. There was also a non-significant trend for women to have worse outcomes than men in our cohort.

The overall mortality rate appeared to be high, at $37.9 \%$. This was despite the introduction of evidencebased therapeutics such as Dexamethasone, Tocilizumab and Remdesevir. A possible explanation for this is that we had a high threshold for admission during the second wave.

There were 41 patients who did not go to ICU despite being on levels of respiratory support that would normally mandate an ICU admission. If one posits that the main value of CPAP is in preventing intubation and protecting ICU capacity, then cHDU has been successful in achieving this goal during wave two, reducing ICU admissions by up to $42 \%$.

High levels of respiratory support, particularly patients receiving the maximum of 30 litres of supplemental oxygen with CPAP, was found to be a very poor prognostic marker. Indeed after 24 hours of such treatment, the only survivors in this group were those who went to ICU. Even of those that went on to invasive ventilation the survivor numbers were still small.

\section{Interpretation}

There is currently no evidence of a survival benefit from CPAP or HFNO in the context of Covid pneumonitis. However, it is thought that non-invasive CPAP may prevent the need for intubation and ventilation in cases where the patient cannot be adequately oxygenated by simple supplemental oxygen.

In our experience, patients with Covid pneumonitis whose gas exchange is sufficiently impaired to require CPAP plus 15 litres of oxygen per minute could not be adequately oxygenated with a standard oxygen 
mask. In some other units, patients reaching this level of support would be considered for ICU admission.

In contrast, our practice has been to provide up to 30 litres of oxygen with CPAP and up to $0.95 \mathrm{FiO}_{2}$ with HFNO. In fact, of our 153 admissions, close to half of our patients (73 or $47.7 \%$ ) were treated with high levels of respiratory support at some point during their stay in cHDU. Of these, 32 ended up going to ICU anyway, but 41 did not. Of the 41 who did not go to ICU, we had only 14 survivors (34.1\%), though the patients who were this unwell and were taken to ICU had similar mortality rates. This certainly suggests a role for high-level respiratory support in an HDU setting as an alternative to invasive ventilation in ICU for some patients.

\section{Limitations}

We need to be cautious in interpreting these findings, since it is likely that there are clinical differences between the ICU and non-ICU groups that are not apparent in the data. It may be that the cohort taken to ICU were at the more severely unwell end of this range, or had level 3 care needs other than respiratory support. However, there does not seem to have been a clear disadvantage in terms of mortality to the cohort treated within CHDU as opposed to ICU, and this suggests that CPAP with high levels of supplemental oxygen may be a reasonable alternative to invasive ventilation in selected patients with Covid pneumonitis.

Although our 153 patients allow us to make some very clear inferences about outcomes based on age and level of respiratory support, this is observational data and there will certainly be confounding variables and selection biases that are not recorded in the data. We should therefore be careful not to over-interpret apparent patterns in the data.

Finally, the design of this study does not allow any definite conclusions to be drawn about the optimal mode of respiratory support in patients with Covid-19 pneumonitis.

\section{Generalisability}

Our results suggest that for patients with severe COVID-19 pneumonitis, CPAP with up to 30 litres per minute of supplemental oxygen is a reasonable alternative to IMV and can protect ICU capacity. For patients who fail to oxygenate at 30 litres per minute, the prognosis is very poor whether they are intubated or not, but at this level we would consider that a trial of CPAP has failed. There is no reason to suspect that these findings would be substantially different in other populations.

\section{Conclusion}

Our data suggests CPAP has a clear role in preventing or delaying ICU admission in patients with severe respiratory failure due to COVID-19. It can be safely delivered with up to 30 litres per minute of supplemental oxygen in a non-ICU setting. Prognosis is poor for those who require high levels of supplemental oxygen with CPAP for a prolonged period of time, with or without subsequent IMV. CPAP should be considered a key tool in alleviating pressure on IMV beds due to COVID-19. 


\section{Abbreviations}

CPAP Continuous Positive Airway Pressure

ICU Intensive Care Unit

IMV Invasive Mechanical Ventilation

cHDU COVID High Dependency Unit

NHS National Health Service

RSU Respiratory Support Unit

HFNO High Flow Nasal Oxygen

AGP Aerosol Generating Procedure

GRI Glasgow Royal Infirmary

\section{Declarations}

\section{Ethics Approval}

This study was approved by the chair of our Regional Ethics Committee (Ref GSH/21/ME/001), and the use of anonymised patient data was granted by the local Caldicott Guardian.

\section{Consent for Publication}

Not applicable.

\section{Availability of Data and Materials}

Datasets used and analysed during this study are available from the corresponding author on request.

\section{Funding}

There was no external funding used.

\section{Competing Interests}

None of the authors have any competing interests to declare.

\section{Author Contributions}

AC collected and analysed the data, and wrote the methods and results sections. SF and LK researched the existing literature and wrote the introduction and discussion sections. PG helped with study design 
and interpretation of results, as well as having editorial input to writing the paper. The authors followed the STROBE checklist for reporting cohort studies when writing this paper.

Acknowledgements

Not applicable.

\section{References}

1. Dong E, Du H, Gardner L. An interactive web-based dashboard to track COVID-19 in real time. The Lancet Infectious Diseases 2020;20:533-4.

2. The RECOVERY Collaborative Group. Dexamethasone in Hospitalized Patients with Covid-19. New England Journal of Medicine 2021;384:693-704.

3. The REMAP-CAP Investigators. Interleukin-6 Receptor Antagonists in Critically III Patients with Covid19. New England Journal of Medicine 2021;384:1491-502.

4. Government U. Coronavirus (COVID-19) in the UK. 2021.

5. Franco C, Facciolongo N, Tonelli R, Dongilli R, Vianello A, Pisani L, et al. Feasibility and clinical impact of out-of-ICU noninvasive respiratory support in patients with COVID-19-related pneumonia. European Respiratory Journal 2020;56:2002130.

6. Walker J, Dolly S, Ng L, Prior-Ong M, Sabapathy K. The role of CPAP as a potential bridge to invasive ventilation and as a ceiling-of-care for patients hospitalized with Covid-19-An observational study. PLOS ONE 2020;15:e0244857.

7. Nightingale R, Nwosu N, Kutubudin F, Fletcher T, Lewis J, Frost F, et al. Is continuous positive airway pressure (CPAP) a new standard of care for type 1 respiratory failure in COVID-19 patients? A retrospective observational study of a dedicated COVID-19 CPAP service. BMJ Open Respiratory Research 2020;7:e000639.

8. Cheung TMT, Yam LYC, So LKY, Lau ACW, Poon E, Kong BMH, et al. Effectiveness of Noninvasive Positive Pressure Ventilation in the Treatment of Acute Respiratory Failure in Severe Acute Respiratory Syndrome. Chest 2004;126:845-50.

9. Alraddadi BM, Qushmaq I, Al-Hameed FM, Mandourah Y, Almekhlafi GA, Jose J, et al. Noninvasive ventilation in critically ill patients with the Middle East respiratory syndrome. Influenza and Other Respiratory Viruses 2019;13:382-90.

10. Zhou F, Yu T, Du R, Fan G, Liu Y, Liu Z, et al. Clinical course and risk factors for mortality of adult inpatients with COVID-19 in Wuhan, China: A retrospective cohort study. The Lancet 2020;395:105462.

11. Dr Ben Messer MAB Ms Pearlene Antoine-Pitterson. BTS/ICS guidance: Respiratory care in patients with acute hypoxaemic respiratory failure associated with COVID-19. 2021.

12. Public Health Scotland. COVID-19 in Scotland. 2021. 
13. Ooms J. Pdftools: Text extraction, rendering and converting of PDF documents. 2021. https://CRAN.R-project.org/package=pdftools.

14. $\mathrm{R}$ Core Team. R: A language and environment for statistical computing. Vienna, Austria: $\mathrm{R}$ Foundation for Statistical Computing 2021. https://www.R-project.org/.

15. CLOPPER CJ, PEARSON ES. THE USE OF CONFIDENCE OR FIDUCIAL LIMITS ILLUSTRATED IN THE CASE OF THE BINOMIAL. Biometrika 1934;26:404-13.

16. Lemmers DHL, Abu Hilal M, Bnà C, Prezioso $C$, Cavallo E, Nencini N, et al. Pneumomediastinum and subcutaneous emphysema in COVID-19: Barotrauma or lung frailty? ERJ Open Research 2020;6. doi:10.1183/23120541.00385-2020

17. Goldman N, Ketheeswaran B, Wilson H. COVID-19-associated pneumomediastinum. Clinical Medicine 2020;20:e91-2.

\section{Figures}

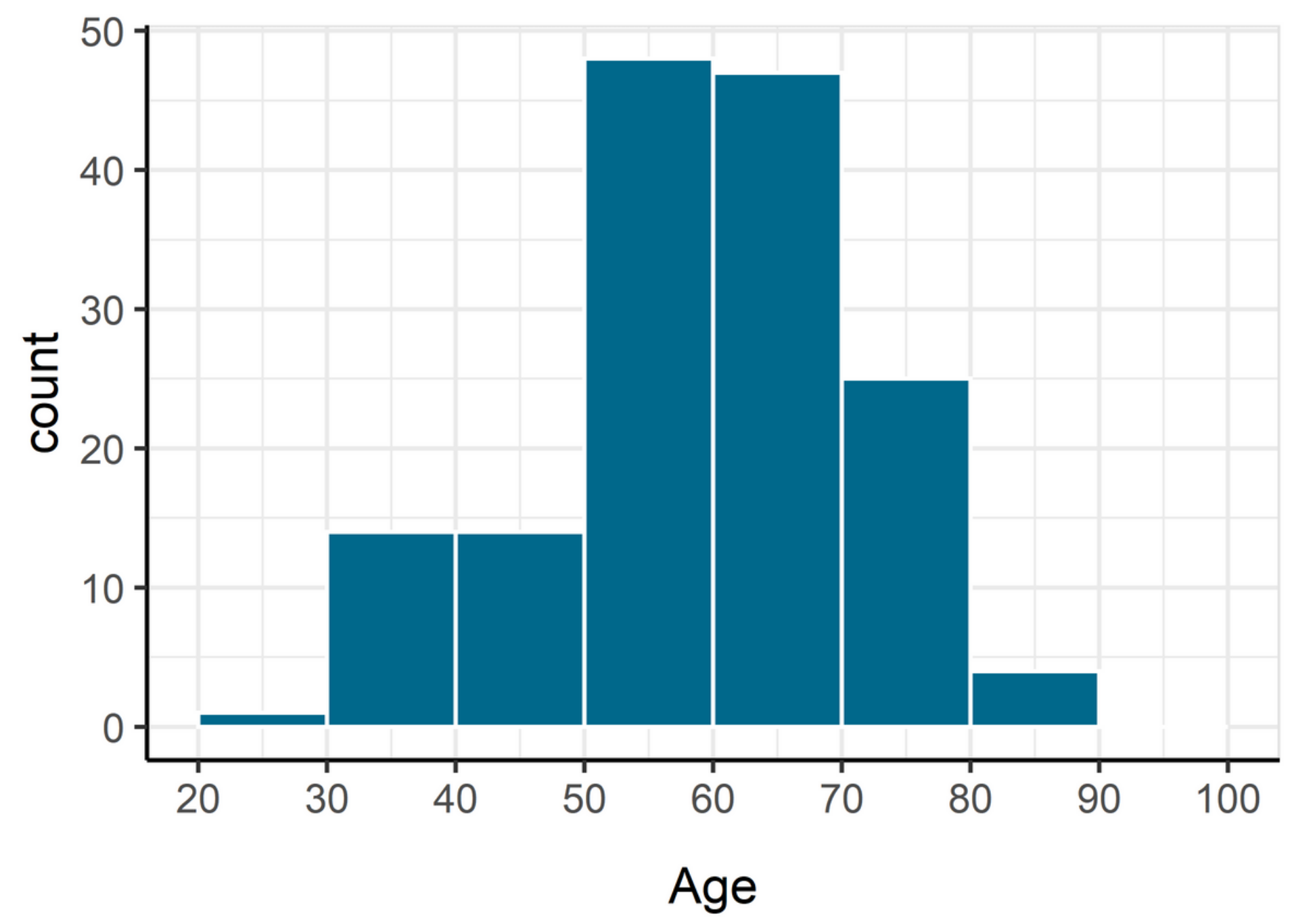

Figure 1 
Age Distribution

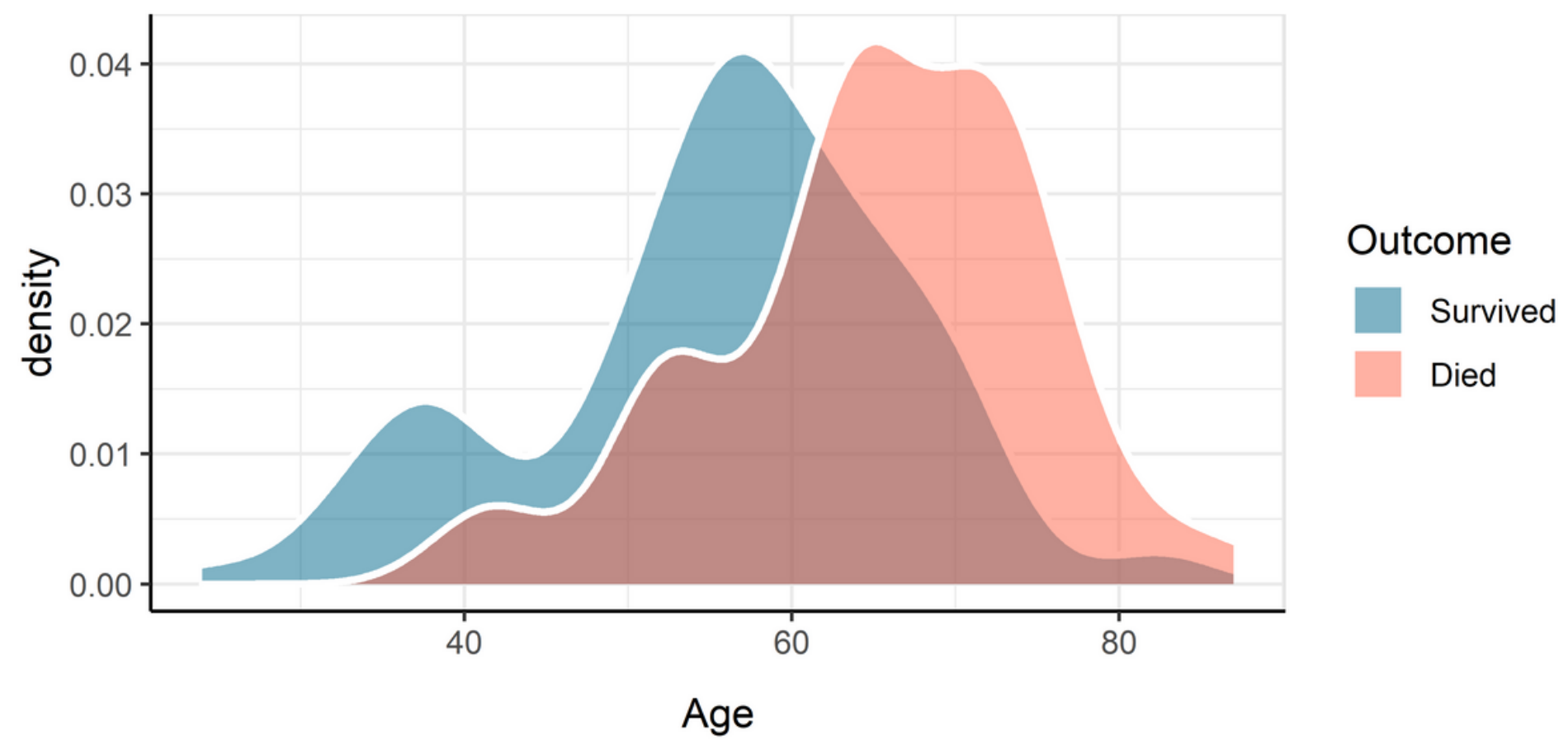

Figure 2

Age density of survivors and non-survivors 


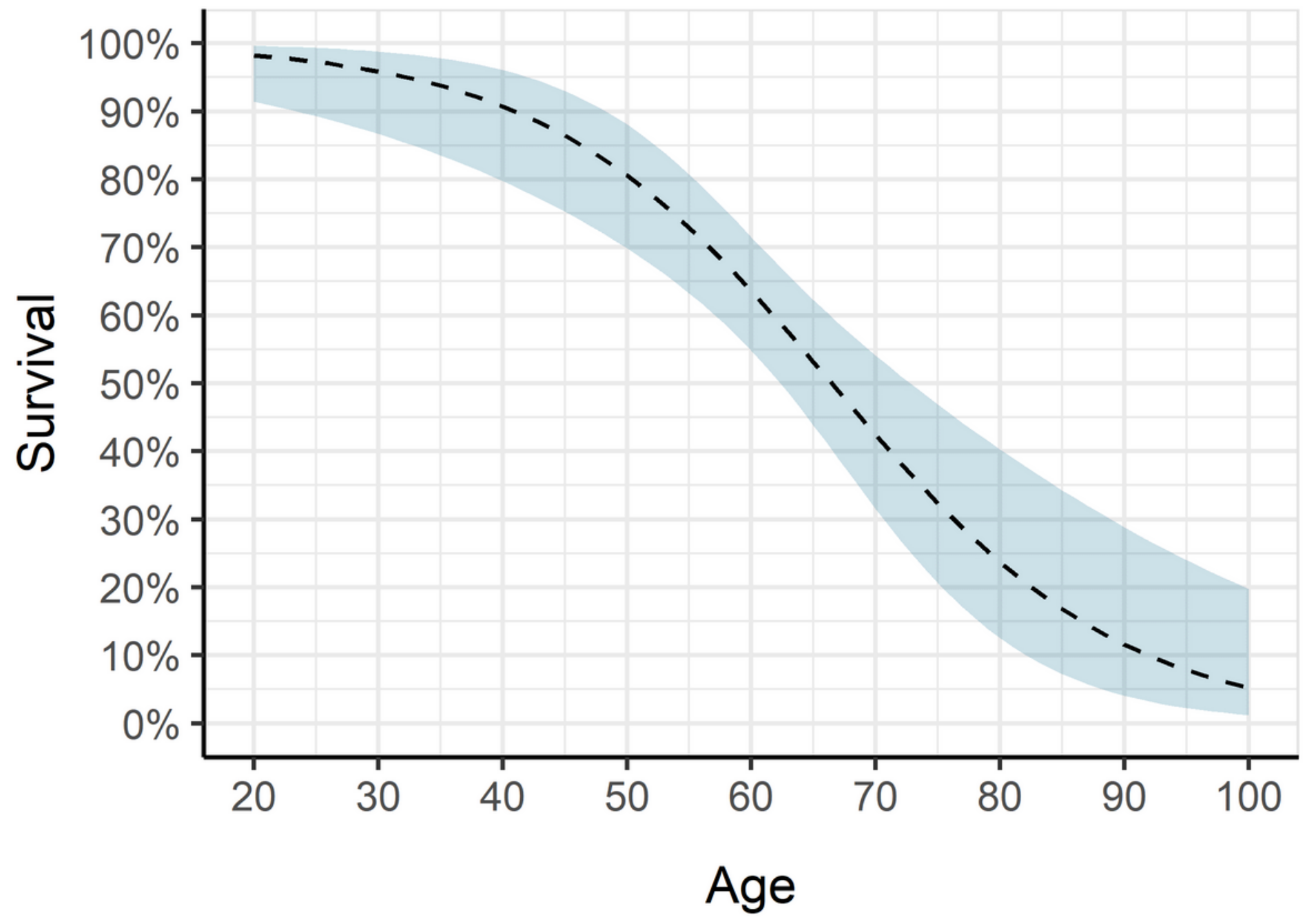

Figure 3

Logistic regression model of association between age and survival with $95 \%$ confidence interval 


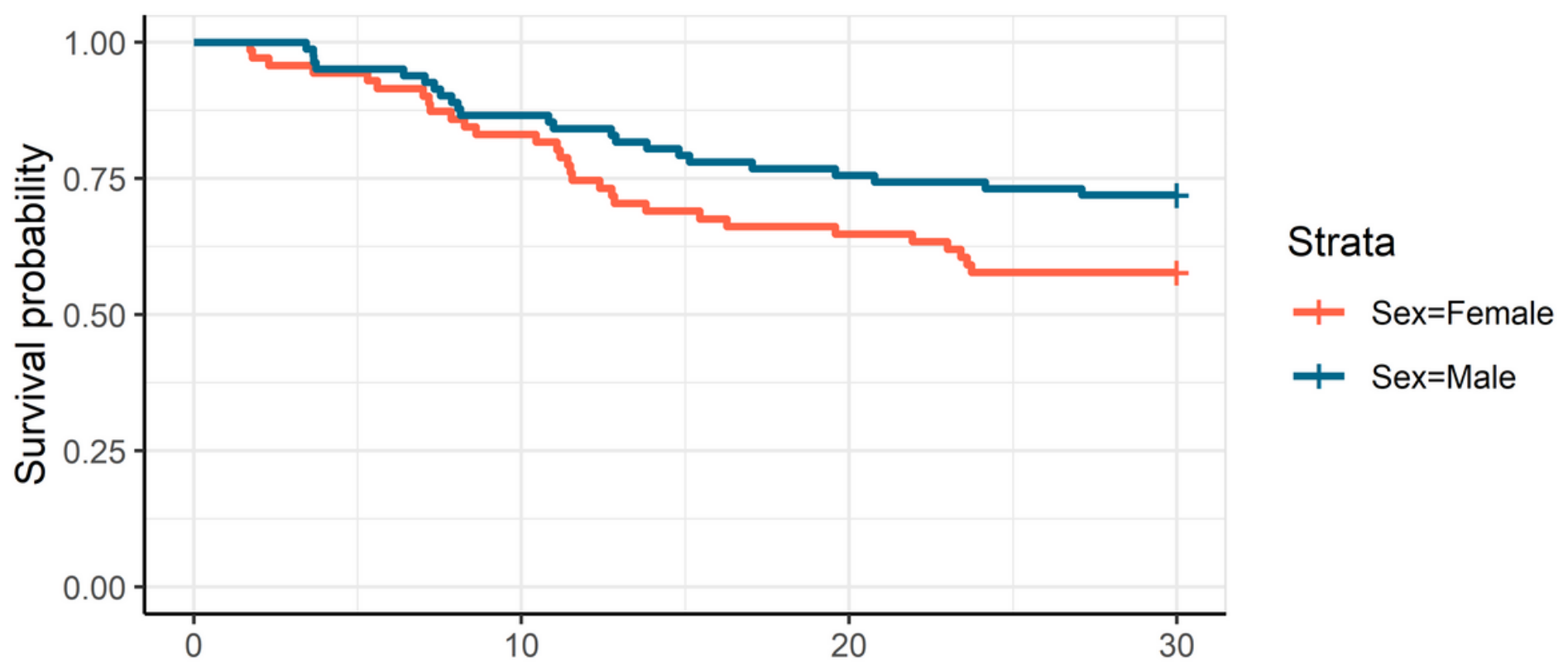

Follow-up time (Days)

Figure 4

Kaplan-Meier survival curves for men and women

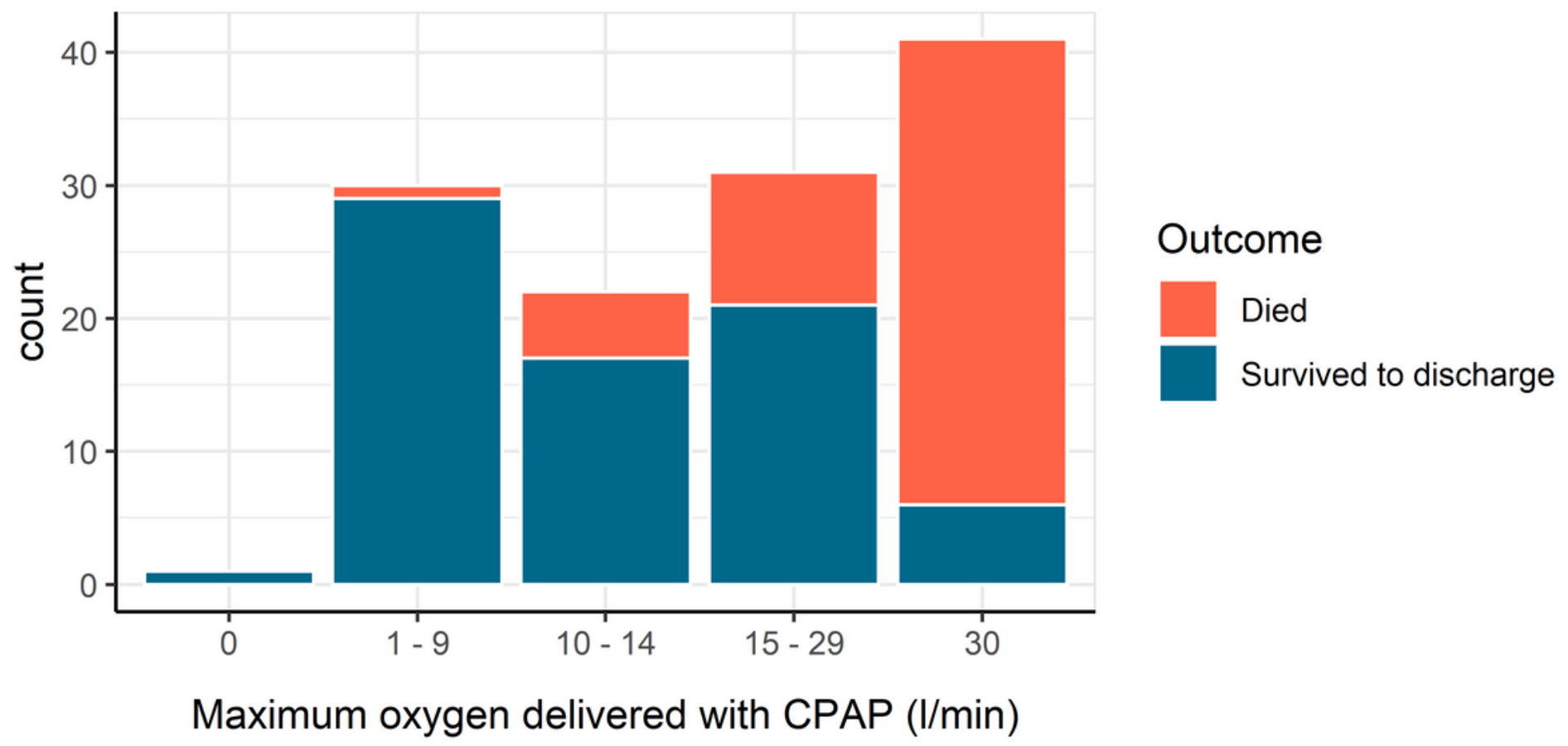

Figure 5

Survival according to maximum supplemental oxygen required with CPAP (absolute numbers) 


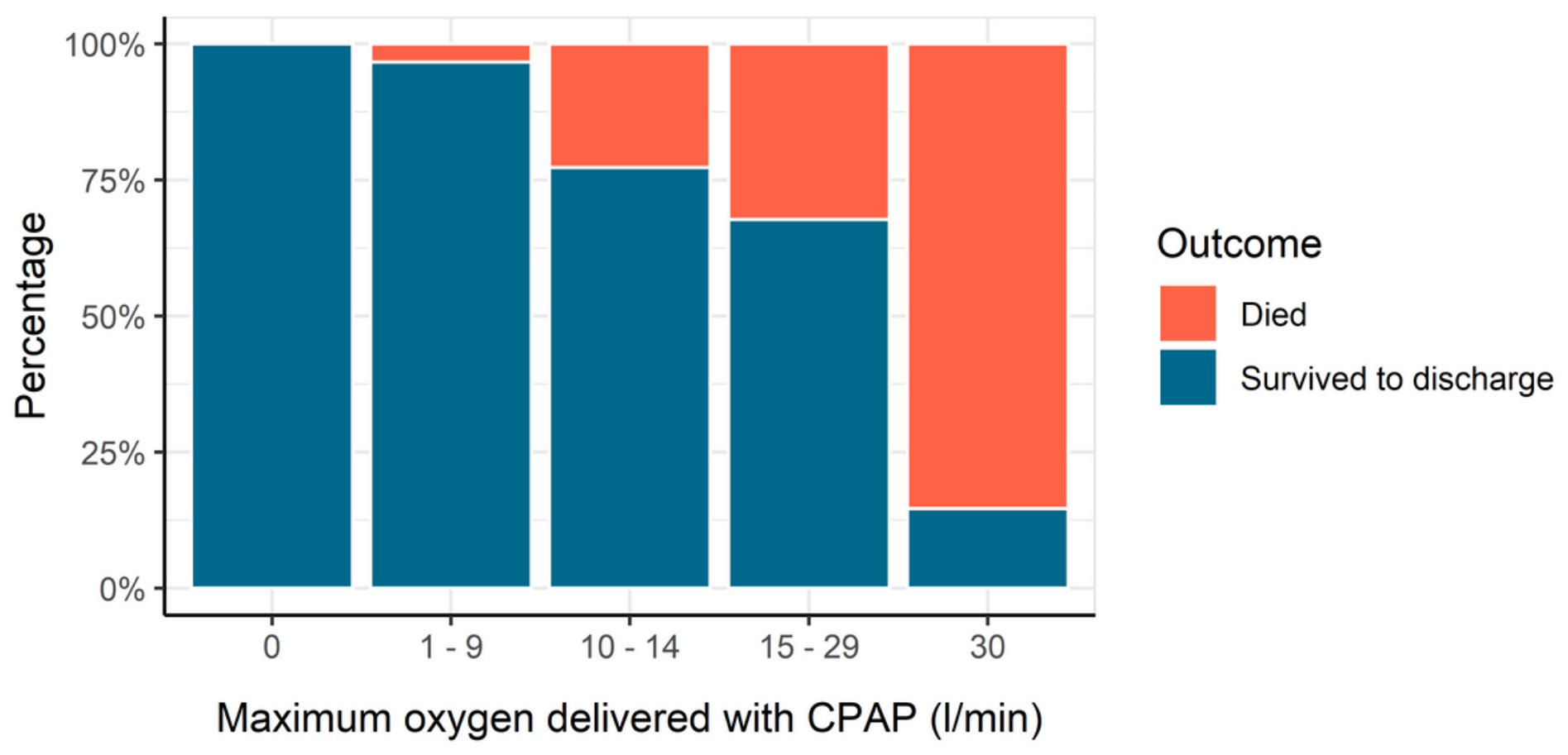

Figure 6

Survival according to maximum supplemental oxygen required with CPAP (as proportion) 


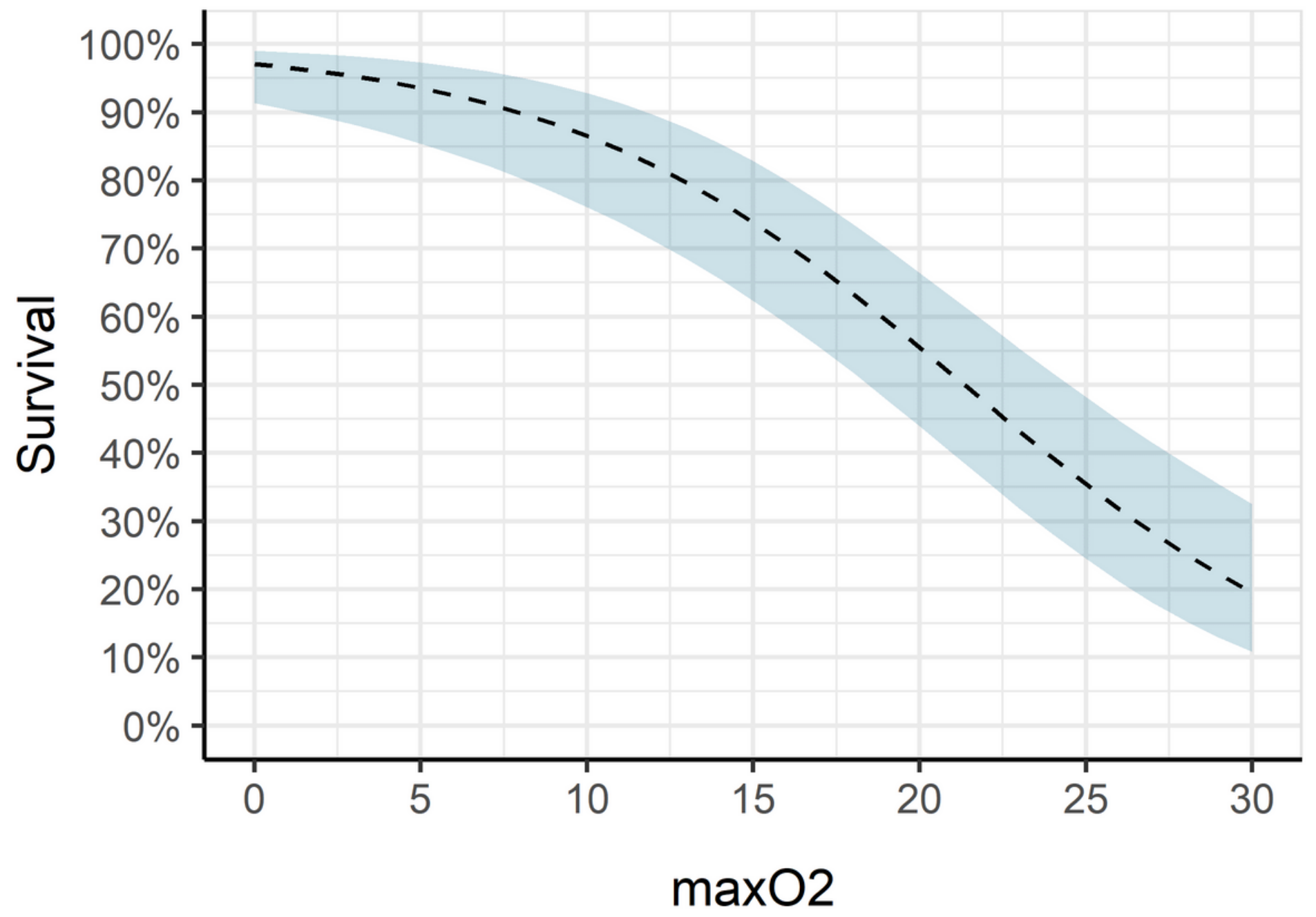

Figure 7

Logistic regression of survival on maximum supplemental oxygen required with CPAP, including 95\% confidence interval 


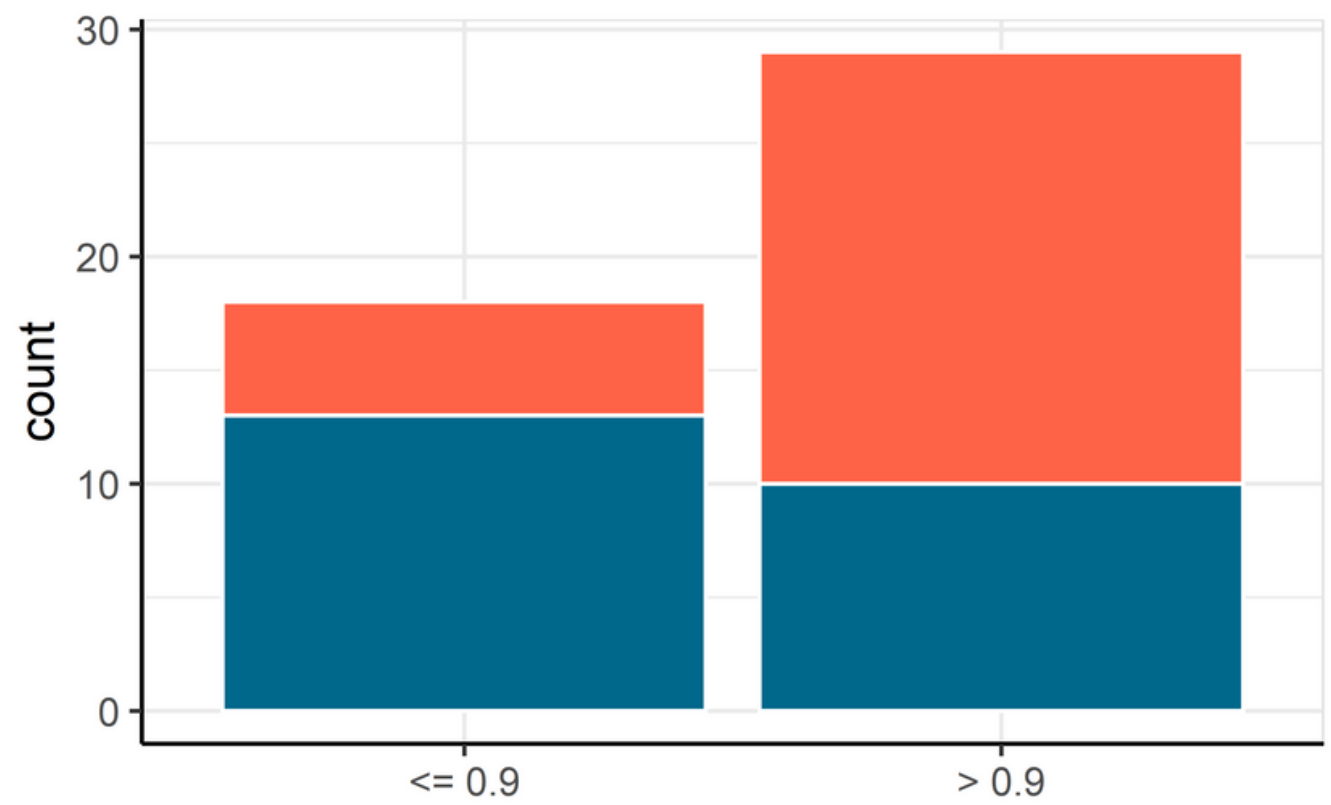

Maximum oxygen delivered with HFNO (FiO2)

\section{Outcome}

Died

Survived to discharge

\section{Figure 8}

Survival according to maximum FiO2 required with HFNO

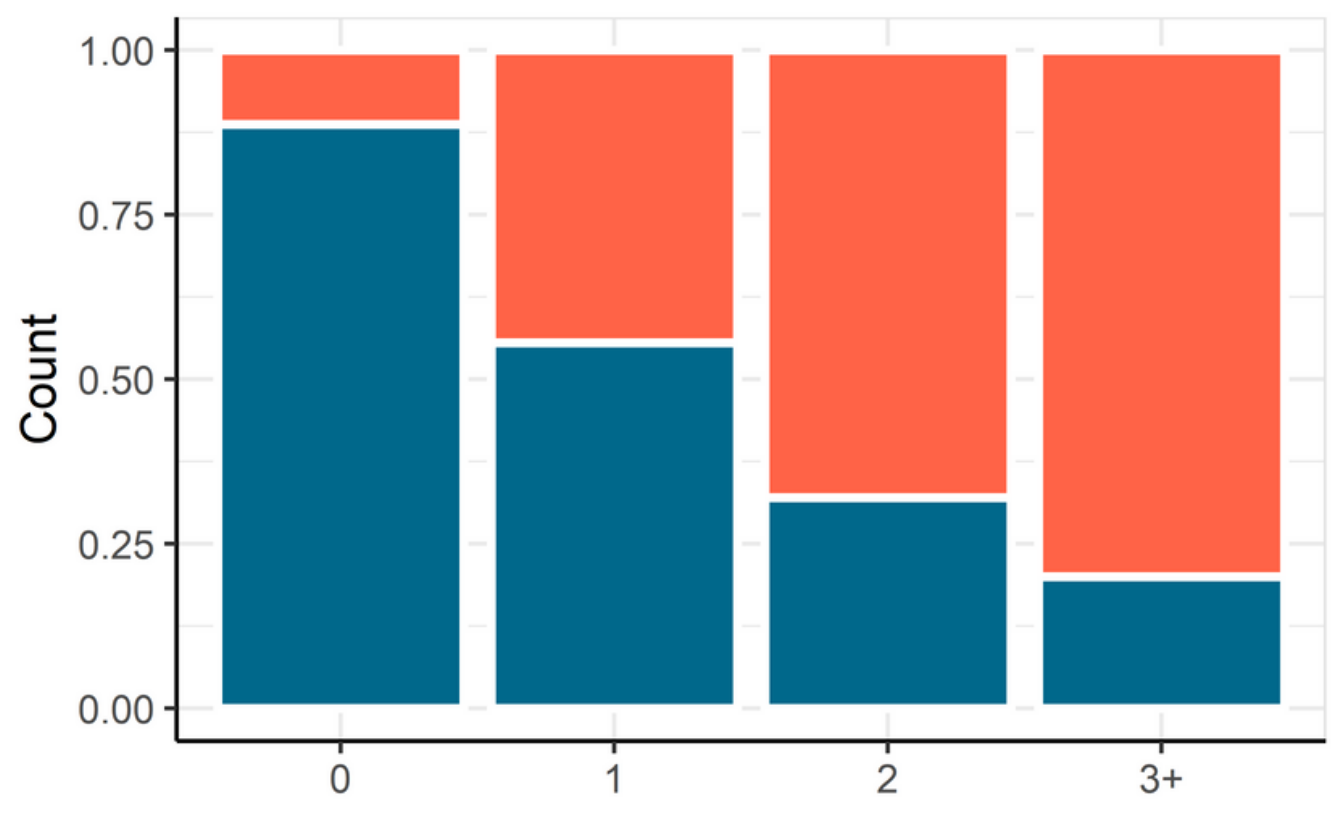

Outcome

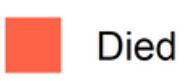

Survived to discharge

\section{Days on high oxygen support}

\section{Figure 9}

Survival according to duration on high respiratory support 